Review Article

\title{
Impact of emerging serotypes on rotavirus immunization
}

\begin{abstract}
Rotavirus infection is a universal disease that commonly affects children below the age of 5 years. The disease causes severe dehydration and may be fatal, especially in the younger age groups. In low socio-economic countries, the median age of infection is 6-9 months, and pre-existing co-morbid conditions, malnutrition, infections etc, make the population more vulnerable. The Rotavirus is a triple layered virus and has the VP4 and VP7 antigens which exist as different serotypes and genotypes. Re-assortment of the G and the P types gives rise to a multitude of viral strains, which vary with demographic variations. G1-4 and P4, P8 are the globally recognized strains. However, there are some new strains that have been noted with increasing frequency in India. These include G9P6, G9P11, G12P6, $\mathrm{G} 12 \mathrm{P} 4$, which show varying degrees of isolation from various parts of our country. While the currently available licensed vaccines have shown dramatic results in decreasing the morbidity and mortality by both homotypic and heterotypic protection in low income countries like Brazil and Mexico where they were introduced in the national immunization schedule as early as 2006-2007, India is yet to introduce Rotavirus vaccines in the national immunization program. Despite the emergence of new disease causing strains, the existing vaccines continue to play a role in providing protection against severe Rotaviral infection in children.
\end{abstract}

Volume I Issue 2 - 2014

\author{
Sumitha Nayak \\ Consultant Pediatrician, The Children?s Clinic, India
}

Correspondence: Sumitha Nayak, Consultant Pediatrician, The Children $\square$ s Clinic, \#4 NTI Colony, 2nd Main Road, 2nd Stage, Raj MahalVilas Extension, Bangalore, India,Tel 91-974213106I, Email delinhong@njau.edu.cn

Received: May 09, 2014 | Published: May 24, 2014

\section{Introduction}

Children below the age of 5 years usually suffer from at least one episode of Rotavirus diarrhea. The median age of infection varies according to the geographical location, but nearly all countries are affected by this virus. According to the 2013 WHO position paper, children between 6-9 months form the median age group in low income countries, while it is a bit later, between 2-5 years in the high income countries. ${ }^{1}$ While the infections may be severe and require hospitalization for children from high income countries, in the low income countries the pre existing co-morbid conditions like underlying infections, malnutrition etc. make the children more susceptible to fatality due to severe Rotavirus disease.

WHO estimates reveal the occurrence of around 420,000-494,000 deaths in 2008 related to Rotavirus gastroenteritis, out of which approximately $90 \%$ occurred in the low income countries of Asia and Africa. ${ }^{1}$ Reports from sentinel hospitals in 35 nations, representing varying geographic areas and economic levels have reported between $34 \%-45 \%$ of diarrheal hospitalizations related to Rotavirus infections. This emphasizes the universality of Rotavirus in causing illness, irrespective of the geographic distribution and the economic status.

\section{Rotavirus strains found in humans}

The Rotavirus belongs to the family Reoviridae which is a triple layered virus, and contains 11 double stranded RNA's in its inner core. ${ }^{2}$. Each segment of the gene codes for either structural proteins or non structural proteins. The outer capsid has two proteins, the VP4 (protease-activated) and VP7 (glycoprotein), which produce a neutralizing response that is serotype specific as well as cross reactive, and is important for protective immunity. The classification of serotypes is based on the varying combinations of the different VP4 and VP7 antigens. There are 12 different VP7 antigens (G-types) and 15 different VP4 antigens (P type) which are found in humans. ${ }^{1}$ The possibility of individual variation of the $G$ and $P$ types has resulted in the usage of a binomial typing. The inner capsid is composed of the VP6 protein, which provides group and sub-group antigenicity, which again is helpful in the classification of the viruses. Most of the human infections are related to the Group A viruses, which may possess subgroup I, II, both or no subgroup antigens. The NSP4 is the non structural protein that contains a toxic peptide region aa114135 , which is of importance as it plays a role in the pathogenesis of diarrhea. ${ }^{2}$ Studies have shown that viral attenuation may be associated with sequence changes in the NSP 4 gene.

Four common Rotavirus genotypes, $\mathrm{G}^{\mathrm{P}} \mathrm{P}^{8}, \mathrm{G}_{2} \mathrm{P}^{4}, \mathrm{G}^{3} \mathrm{P}^{8}$ and $\mathrm{G} 4 \mathrm{P}^{8}$ have been found to be in circulation globally along with certain other less common serotypes. ${ }^{3}$ Hence most of the cases of infections have been found to be related to these viral genotypes. However, some studies conducted as early as 1992 and 1993 reported the presence of a novel Rotavirus strain, with the prototype 116E New Delhi with a G9P ${ }^{11}$ specificity, that had been isolated from newborns in New Delhi, India. ${ }^{4}$ They also reported another novel strain G9P, ${ }^{6}$ which had completely replaced G9P. ${ }^{11}$ in some hospital isolates. It was postulated that the $\mathrm{G} \mathrm{P}^{6}$ strain arose due to re-assortment, while subsequent co-circulation of $\mathrm{G}^{1} \mathrm{P}^{11}$ along with $\mathrm{G}^{9} \mathrm{P}^{6}$ was also found. Another unusual strain, G3P. ${ }^{9}$ was reported from Malaysia, and this strain was closely related to a raccoon dog Rotavirus strain. ${ }^{5}$ This $\mathrm{G} \mathrm{P}^{9}$ genotype was also isolated from some children in Italy, where the genome sequencing showed that they were composed of genes from human, bovine as well as feline strains. Thus, it was postulated that these animal strains which entered and adapted to the human environment caused limited disease, rather than exist as a complete strain. Thus many combinations $\mathrm{G}$ and $\mathrm{P}$ types are possible and epidemiological data has shown the occurrence of a wide range of diverse Rotavirus strains in Africa, including the common G1 type to the less common G8, G9, G10 and G12 types. ${ }^{6}$ In Malawi, G12 was the predominant circulating strain noted during the study by Steele et al. ${ }^{6}$ and strains with $\mathrm{P}^{6}$ genotype were common in young African children with symptomatic Rotavirus infection. Brazil first reported the presence of G9 serotype in 2000, and in the year 2002 it accounted for $46.9 \%$ of the typed isolates. ${ }^{7}$ Of these, The G9P ${ }^{8}$ type 
was the most prevalent (75.7\%), followed by G9P and G9P. ${ }^{6}$ Reports of studies done in Bangladesh between 2004 and 2006revealed $\mathrm{G}_{2} \mathrm{P}^{4}$ as the highest isolate in $36.7 \%$ cases followed by $\mathrm{G}^{8} \mathrm{P}^{8}$ in $10 \%$ cases. ${ }^{8}$ There were also reports of G12P. ${ }^{6}$ and G12P, ${ }^{4}$ isolated for the first time in that country. Hospital based studies conducted between 2005 and 2009 in India, showed an increase in the incidence of G12P. ${ }^{6}$ infections from $8 \%$ to $39 \%$ in Northern India and from $8 \%$ to $34 \%$ in Western India , while G9 was isolated in $13 \%$ of cases. Studies done in South Korea over five Rotavirus seasons, showed the presence of 18 strains of G9 as causative agents, all related to G9P. ${ }^{8,9}$ While the G12 strain was initially isolated from Philippines in the year 1987, it has become increasing prevalent worldwide. Similar to the emergence of the G9 strain, the G12 strain has possibly emerged due to genetic re-assortment into a strain that is well adapted to the human gastrointestinal tract. The possibility of the origin of these strains from the porcine Rotavirus has been described due to the close G9 and G12 strain relationship between human strain and porcine strain. Strains of other P-G combinations, which would normally be considered unusual, constitute almost $4.9 \%$ of all isolates. ${ }^{10}$. Rotavirus strains carrying $\mathrm{G} \mathrm{P}^{4}, \mathrm{G} 2 \mathrm{P}^{8}, \mathrm{G}^{2} \mathrm{P}^{4}, \mathrm{G} 4 \mathrm{P}^{9}, \mathrm{G}^{4} \mathrm{P}^{4}, \mathrm{G}^{2} \mathrm{P}^{4}, \mathrm{G}^{2} \mathrm{P}^{10}, \mathrm{G} 12 \mathrm{P}^{6}$ and $\mathrm{G}_{12} \mathrm{P}^{9}$ specificity may indicate naturally occurring reassortants among various human Rotavirus genotypes. Rotaviruses undergo constant genetic variation via point mutations of antigenic drift, genetic reassortment or antigenic shift, genomic rearrangement or intragenic recombination, resulting in the emergence of new strains. ${ }^{10}$ However, unusual Rotavirus $\mathrm{P}-\mathrm{G}$ combinations may result from mixed Rotavirus infections. Cyclic strain variations have been known to occur every 3-5 years, wherein the Rotavirus strain predominance patterns undergo variations. ${ }^{11}$ Together with the re-assortments that occur from animal strains, there could be a wide variability in the Rotavirus types that cause infections in children.

\section{Protective immunity and circulating vaccine strains}

Protection against infection is mediated both by humoral as well as cellular immunity, and the serological response is directed against the specific serotype which is a homotypic response. ${ }^{1}$ With repeated infections, the risks of severity progressively decreases and the subsequent infections may be due to other $G$ types. The protection provided by immune responses to VP4 and VP7 proteins is an important correlation for decreasing disease severity. ${ }^{1}$. In a study conducted by Gladstone et al. ${ }^{12}$ in India, it was reported that in children with multiple infections of Rotaviral diarrhea, the severity did not decrease between the first and the second infections, but only reduced between the second and third infections. According to WHO reports, the risk of severe disease continued after several reinfections. ${ }^{1}$ This could point towards the necessity to provide adequate protection against severe Rotavirus infection for at least the initial two episodes, which could be due to a different $\mathrm{G}$ serotypes. The common serotypes isolated in the Gladstone et al. ${ }^{12}$ study were $\mathrm{G}^{8} \mathrm{P}^{8}$ in $15.9 \%$, $\mathrm{G}^{2} \mathrm{P}^{4}$ in $13.6, \mathrm{G} 10 \mathrm{P}^{11}$ in $8.7 \%, \mathrm{G}^{8} \mathrm{P}^{8}$ in $7.2 \%$, while $\mathrm{G}^{\mathrm{P}} \mathrm{P}^{4}$ was isolated in $4.4 \%, \mathrm{G} 10 \mathrm{P} 4$ in $1.75, \mathrm{G} 9 \mathrm{P} 4$ in $1.5 \%, \mathrm{G} 12 \mathrm{P} 6$ in $1.1 \%$ and $\mathrm{G}^{\mathrm{P}} \mathrm{P}^{6}$ in 0.65 of cases. The G10P. ${ }^{11}$ serotype was found mainly in neonates and was asymptomatic in most cases. Another study conducted in India between 2004-2007 by Sharma et al. ${ }^{13}$ revealed the increased frequency of G12 and G9 strains while the G1 strain was decreased and the G3 and G4 were rarely isolated. This study also showed that the bulk of disease, between $40-60 \%$ of all Rotavirus infections annually was caused by the combination of G1P8, G2P4, G9P8 and G12P6 strains combined. The study conducted by Bahl et al. ${ }^{14}$ which covered South Delhi population and hospitals showed interesting results. While G1 and G9 strains were commonly isolated during the winter months and G1 and G2 between July-October, the G9, G1 and
G2 strains were also common during the summer months. Mixed G types infection was identified in $10 \%$, and only G1P8, G2P4, G9P8 and G9P6 were commonly isolated, along with G1P6 and G3P6 which were frequently identified, though not present globally. This study also showed that the mean severity of the diarrhea and dehydration was higher in children who suffered from G1 strain infection, than in those who had other strains, and these children needed hospitalization and aggressive treatment. The P type was not co-related to the clinical severity. Thus, we have to look at providing immunization protection against a variety of strains, and vaccines which can provide a broad spectrum of immunity, as against strain specific immunization. The vaccine must be effective against the G1 strain which is the cause of infection with great clinical severity associated with severe dehydration.

\section{Does immunization decrease mortality?}

The target for Rotavirus vaccination was to decrease the morbidity due to infection in the developing world and the morbidity due to Rotavirus in the developed world. Before we undertake to introduce any vaccine in the community, it is essential to ascertain its role in improving the quality of life of the target population. It is only after confirming this, can the economics and feasibility of vaccine coverage be considered. Mexico and Brazil have a large population of underfives and the morbidity and mortality in this age group due to diarrhearelated complications has been comparable with that of any developing country in the world. In a study conducted to estimate the diarrhea related deaths in children below 5 years of age from 2003-2006, the maximal numbers $(67 \%)$ occurred in children below 11months, while $23 \%$ occurred between $12-23$ months and $10 \%$ of deaths occurred in those between 24-59 months of age. ${ }^{14}$ The proportion of hospitalizations attributable to the Rotavirus infections seemed to have increased between 2000-2004, compared with other causes of severe childhood diarrhea. ${ }^{14,15}$ This probably co-related with improvements in sanitation and hygiene that impacted the transmission of bacteria and other parasitic agents which are transmitted through contaminated food and water, and for which the person to person transmission can occur. However, these methods do not affect Rotavirus transmission as was noted by the higher numbers of Rotaviral infection during the winter months, and where the administration of ORS is hampered due to persistent vomiting and large stool volumes. According to estimates from WHO, approximately 3100 diarrhea related deaths occurred annually in under -5 's in Mexico due to Rotaviral disease. ${ }^{15}$ A monovalent Rotavirus vaccine was introduced in a graded manner into the routine immunization program of Brazil in the year 2005..$^{7}$ and in Mexico, in the year2006, and by the year 2007, this vaccine covered all the age eligible Mexican children. They were administered 2 doses of the vaccine at 2 and 4 months of age. The results of the Mexican immunization program was noticeable in the year 2008, when the diarrhea related deaths declined maximally in those infants below 11 months, from $61.5 / 100,000$ in 2003-2006 to $36.0 / 100,000$ in $2008 .^{15}$ The $12-23$ months age group also showed considerable decline from, $21.1 / 100000$ to $15.0 / 100000$, while in the 24-59 months the decline was not significant with the relative rate being reduced from $2.9 / 100000$ to $2.7 / 100000$. Thus the impact of the immunization program was evident in the subsequent Rotaviral season, with the maximal impact in the target group and some amount of protection in the other ages, which could be due to the occurrence of the herd immunity. However, the emerging strains pose a challenge for the adequacy of coverage by the currently available type specific vaccines. The question of the feasibility of introducing strain specific vaccines and its capability to provide adequate protection against all Rotavirus infections needs to be carefully debated. 


\section{Role of the currently available vaccines in the scenario of emerging new strains}

Currently available vaccines are live oral attenuated Rotavirus strains of human and/or animal origin, which replicate in the human intestine to provide immune responses. ${ }^{1}$ These vaccines are the monovalent (RV1) vaccine and the pentavalent human-bovine recombinant vaccine (RV5), both of which are available internationally. The RV1 is derived from the RIX4414 strain which has been attenuated by serial passage in Vero cells, containing attenuated G1P Rotavirus particles, and is recommended in two doses after the age of 6 weeks, and completion of course by 24 weeks. The RV 5 vaccine is derived from the human and bovine WC3 parent Rotavirus strains with four of the reassortants that express G1, G2, G3 or G4 from the human strain and $\mathrm{P}^{5}$ from the bovine strain, while the fifth reassortant expresses $\mathrm{P}_{1} \mathrm{~A}^{8}$ from a human strain with $\mathrm{G} 6$ from a bovine parent strain. ${ }^{1}$ Cochrane reviews have reiterated the efficacy of both RV1 and RV5 vaccines against severe Rotavirus gastroenteritis (RVGE) in all the WHO sub regions that have child and adult mortality ranging from very low to high or very high mortality rates. ${ }^{16}$ The Cochrane metaanalyses show efficacy ranges of $40-60 \%$ in regions with high child mortality. Rotavirus vaccination has decreased the disease burden within a few years of vaccine introduction, as has been noted in Brazil and Mexico, and also has decreased the magnitude of the annual seasonal infections in the high income countries like USA, Finland etc. ${ }^{1,17}$ Apart from these, there are several candidate live-attenuated Rotavirus vaccines that are developed in different parts of the world. One such vaccine is based on the Rotavirus strain $116 \mathrm{E}\left(\mathrm{G} \mathrm{P}^{11}\right)$, which has shown good immunity in young Indian children. Some other manufacturers are also in the process of developing a vaccine based on the UK bovine-human Rotavirus reassortant strains. These vaccines are targeted to provide protection against the locally prevailing strains. However, natural secular strain changes have been noted even in the prevaccine era. Hence, once the strain specific vaccines are introduced, the likelihood of strain replacement has to be considered, which is likely due to strain reassortment. According to Miles et al. ${ }^{18}$ significant shifts in Rotavirus strain diversity have occurred in the past three decades, hence it is important to understand the strain diversity profile before vaccine introduction. While neither of the currently available licensed vaccines contain G9, G12, P. ${ }^{4}$ or $\mathrm{P}^{6}$ components, they have both shown broad heterotypic protection in various pre-licensure and post-licensure studies. ${ }^{9}$ A large study lasting 27 years was conducted in Brazil and covering both the pre and post vaccine periods. While the majority of cases in this study $(42.9 \%)$ belonged to the G1 strain, there was a considerable number of G2 strains causing disease during the period 2006-2008. ${ }^{19}$ This was considered to be due to the phenomenon of continental phenomenon, as it was noted in other Latin American countries and Bangladesh, where the vaccine was not yet introduced. It may be inferred that the newer strains that have been found to be associated with disease are emerging due to cyclical strain changes, and the older strains could be co-existent with the newer emerging ones. As India has such vast geographic variability, along with Rotavirus strain variations, ${ }^{18}$ it would be pertinent to consider vaccines that can cover strain diversity and cyclical changes in the prevailing serotypes.

Studies from Brazil have shown $\mathrm{G}_{2} \mathrm{P}^{4}$ as an important disease causing serotype, beside the other circulating strains. However, the vaccine utilized for Rotaviral immunization was the monovalent RV1 vaccine that contained the totally heterotypic serotype $\mathrm{G}^{8} \mathrm{P}^{8}$ strain. Results of post vaccine studies have shown a persistent reduction of diarrhea deaths and diarrhea related hospitalizations in all regions and age groups in Brazil. ${ }^{17}$ Trials already completed in Malawi with the licensed monovalent $\mathrm{G}^{8} \mathrm{P}^{8}$ containing vaccine have shown vaccine efficacy, in addition to the homotypic G1P, ${ }^{8}$ against the heterotypic G12P ${ }^{6}$ strain. ${ }^{9}$ With the complexity of Rotavirus epidemiology and multiple $\mathrm{G}$ and $\mathrm{P}$ serotypes which can circulate simultaneously in a given population, the ability of the Rotavirus vaccine to generate heterotypic immunity remains critical. ${ }^{10}$ While the role of single strain vaccine in protection against heterotypic disease has been strongly debated, the results from Brazil have reiterated the strong heterotypic cross protection that resulted in the decline in diarrhea related disease. ${ }^{17}$ Reports have suggested that there is no need, unlike the Influenza vaccine, to periodically update the composition of the vaccine, as the phylogenies of VP7 genes do not exhibit strong patterns of drift, as well as the fact that the mutation rates are lower, owing to the double stranded nature of the virus. ${ }^{3}$

Collectively, the currently available licensed vaccines should continue to be efficacious despite the changing scenario and emerging Rotavirus strains.

\section{Conclusion}

Rotavirus infection is a worldwide phenomenon, and is responsible for a high rate of morbidity and mortality. Although children between 6-9 months form the median age group in low income countries, it occurs a bit later, between 2-5 years in the high income countries. While the infections may be severe and require hospitalization for children from high income countries, in the low income countries, the pre existing co-morbid conditions like underlying infections, malnutrition etc. make the children more susceptible to fatality due to severe Rotavirus disease. Although G1-4, $\mathrm{P}^{4}$ and ${ }^{8}$ are the common global serotypes, currently there are newer strains that have been found to be responsible for infections in various parts of the world..$^{12}$ In India, the emergence of the G9 and G11 strains has been noted with rising frequency. ${ }^{13}$ While the currently available licensed Rotaviral vaccines are not homotypic for these emerging strains, studies have shown their significant efficacy in decreasing the occurrence of diarrhea related deaths. Based on the socio economic status of the population the single strain RV1 vaccine has shown $85 \%$ protective efficacy against severe Rotavirus disease in Latin America, $71-76 \%$ efficacy in the middle income settings of South Africa and ElSalvador and $49 \%$ efficacy in the low income setting of Malawi. Similar gradients have been observed with the RV5 vaccine in clinical trials and post licensure evaluations. ${ }^{17}$ Thus, while the emerging strains of G9 and G12 need to be specifically targeted due to the cyclical strain shifts that occur, the prevailing strains, except G3 and G4 whose incidence has decline remain to be tackled as disease causing entities. ${ }^{19}$ It is essential to ensure that the Rotavirus vaccines are efficacious against all common circulating Rotavirus strains, and continued strain surveillance will be important. ${ }^{20}$ Vaccines that provide both homotypic as well as heterotypic strain coverage will remain a cost effective and an essential part of the strategy to decrease morbidity and mortality related to Rotavirus diarrhea.

\section{Acknowledgments}

None.

\section{Conflicts of interest}

The authors have no conflict of interests related to this publication and have not received any grants.

\section{Funding}

None. 


\section{References}

1. World Health Organization (WHO). Weekly epidemiological record (WER). 2013;88(5):49-64.

2. Griffin DD, Nakagomi T, Hoshino $Y$, et al. Characterization of nontypeable rotavirus strains from the United States:identification of a new rotavirus reassortant $(\mathrm{P} 2 \mathrm{~A}[6], \mathrm{G} 12)$ and rare $\mathrm{P} 3[9]$ strains related to bovine rotaviruses. Virology. 2002;294(2):256-269.

3. Pitzer VE, Patel MM, LopmanBA, et al. Modelling rotavirus strain dynamics in developed countries to understand the potential impact of vaccination on genotype distributions. Proc Natl Acad Sci USA. 2011;108(48):19353-19358

4. Das BK, Gentsch JR, Cicirello HG, et al. Characterization of rotavirus strains from newborns in New Delhi, India. J Clin Microbiol. 199432(7):1820-1822.

5. Ch'ng LS, Lee WS, Kirkwood CD. Rare rotavirus strains in children with severe diarrhea, Malaysia. Emerg Infect Dis. 2011;17(5):948-950.

6. Steele AD, Neuzil KM, Cunliffe NA, et al. Human rotavirus vaccine Rotarix provides protection against diverse circulating rotavirus strains in African infants:a randomized controlled trial. BMC Infectious Diseases. 2012;12:213.

7. Carmona RCC, Timenetsky MCST, Morillo SG, et al. Human rotavirus serotype G9, Sao Paulo, Brazil, 1996-2003. Emerg Infect Dis. 2006;12(6):963-968.

8. Paul SK, Ahmed MU, Hossain MA, et al. Molecular characterization of group A human rotavirus among hospitalized children and adults in Bangladesh:finding of emerging G12 strain. Mymensingh Med J. 2010;19(1):16-26.

9. Kang G, Desai R, Arora R, et al. Diversity of circulating rotavirus strains in children hospitalized with diarrhea in India, 2005-2009. Vaccine. 2013;31(27):2879-2883.

10. Santos N, Hoshino Y. Global distribution of rotavirus serotypes/ genotypes and its implication for the development and implementation of an effective rotavirus vaccine. Rev Med Virol. 2005;15(1):29-56.
11. Than VT, Kang H, Lim I, et al. Molecular characterization of serotype G9 rotaviruses circulating in South Korea between 2005 and 2010. J Med Virol. 2013;85(1):171-178.

12. Gladstone BP, Ramani S, Mukhopadhya I, et al. Protective effect of natural rotavirus infection in an Indian birth cohort. $N$ Engl J Med. 2011;365(4):337-346.

13. Sharma S, Ray P, Gentsch JR, et al. Emergence of G12 rotavirus strains in Delhi, India, in 2000 to 2007. J Clin Microbiol. 2008;46(4):13431348.

14. Bahl R, Ray P, Subodh S, et al. Incidence of severe rotavirus diarrhea in New Delhi, India, and $\mathrm{G}$ and $\mathrm{P}$ types of the infecting rotavirus strains. $J$ Infect Dis. 2005;192(Suppl 1):S114-S119.

15. Richardson V, Hernandez-Pichardo J, Quintar-Solares M, et al. Effect of rotavirus vaccination on death from childhood diarrhea in Mexico. $N$ Engl J Med. 2010;362:299-305.

16. Soares-Weiser $\mathrm{K}$, Maclehose $\mathrm{H}$, Bergman $\mathrm{H}$, et al.Vaccines for preventing rotavirus diarrhoea:vaccines in use. Cochrane Database Syst Rev. 2012;11:CD008521.

17. do Carmo GMI, Yen C, Cortes J, et al. Decline in diarrhea mortality and admissions after routine childhood rotavirus immunization in Brazil:a time-series analysis. PLoS Med. 2011;8(4):e1001024.

18. Miles MG, Lewis KD, Kang G, et al. A systematic review of rotavirus strain diversity in India, Bangladesh, and Pakistan. Vaccine. 2012;30(Suppl 1):A131-A139.

19. Oliviera A, Mascarenhas JDP, Soares LS, et al. Rotavirus serotype distribution in northern Brazil trends over a 27 year period pre and post national vaccine introduction. Trials in Vaccinology. 2012;1:4-9.

20. Phua KB, Lim FS, Lau YL, et al. Rotavirus vaccine RIX4414 efficacy sustained during the third year of life: A randomized clinical trial in Asian population. Vaccine. 2012;30(30):4552-4557. 